\title{
Reply to the comments of Dr. Quarato et al.
}

\author{
Ana Pičuljan ${ }^{1} \cdot$ Marko Šustić ${ }^{2}$ Alan Šustić $c^{1,3}$
}

Received: 6 February 2020 / Accepted: 12 February 2020 / Published online: 15 February 2020

(c) Springer Nature B.V. 2020

We appreciate the interest of Dr. Quarato et al. in our manuscript investigating inter-observer and intra-observer agreement in the estimation of the exact number of B-lines between observers with three different levels of experience and by the software algorithm in real-time point-of-care ultrasound. However, we should comment on certain points of their letter.

Firstly, the concern of Quarato et al. regards our claim that the cause of B-lines is "interstitial edema" suggesting that it is not always the case, while in fact we clearly emphasize that “ . .. this artifact of various etiologies, although most commonly associated with increased interstitial edema, has also been detected in different interstitial lung diseases".

Secondly, although Quarato et al. claim that some study did not find a significant difference in the number of B-lines between patients with acute pulmonary edema and those with other pulmonary disease, several important recent researches highlighted the clinical significance of the exact numbering of B-lines $[1,2]$. Hence, Ferre et al. find an increase of six Delta-B-line as the threshold value to diagnose weaning-induced pulmonary edema, Miglioranza et al., counting B-lines in predicting of heart decompensation, find two threshold points; B-lines number $\geq 30$ identified a group at high risk of pulmonary edema and number of B-lines $<15$ indicated patients without a risk of pulmonary edema. In last few years, similar results about clinical significance of the

This reply refers to the comment available online at https://doi. org/10.1007/s10877-020-00484-0.

\section{Alan Šustić}

alan.sustic@uniri.hr

1 Department of Anesthesiology, Reanimatology, Intensive Care and Emergency Medicine, Faculty of Medicine, University of Rijeka, Rijeka, Croatia

2 Department of Histology and Embryology, Faculty of Medicine, University of Rijeka, Rijeka, Croatia

3 Department of Clinical Medical Science II, Faculty of Health Studies, University of Rijeka, Rijeka, Croatia
B-line counting were found by Enghard et al., Tardella et al. and others $[3,4]$.

Thirdly, respecting the fact that the number of B-line partly depends on the ultrasound scan setting, we (the software included) used the same ultrasound machine and the probe with same frequency in all patients during the study.

Last but not least, we cannot entirely agree with the conclusion of Quarato et al. Our opinion is that the B-lines counting has a certain clinical relevance today and, with a better quantification and objectification with a software algorithm, this will be even more relevant in the future.

\section{References}

1. Ferre A, Guillot M, Richard C, Teboul JL, Monnet X. Lung ultrasound allows the diagnosis of weaning-induced pulmonary oedema. Intensive Care Med. 2019;45:601-8. https://doi. org/10.1007/s00134-019-05573-6.

2. Miglioranza MH, Picano E, Badano LP, Sant'Anna R, Rover M, Zaffaroni F, Sicari R, Kalil RK, Leiria TL, Gargani L. Pulmonary congestion evaluated by lung ultrasound predicts decompensation in heart failure outpatients. Int J Cardiol. 2017;240:271-8. https ://doi.org/10.1016/j.ijcard.2017.02.150.

3. Enghard P, Rademacher S, Nee J, Hasper D, Engert U, Jörres A, Kruse JM. Simplified lung ultrasound protocol shows excellent prediction of extravascular lung water in ventilated intensive care patients. Crit Care. 2015;19:36. https://doi.org/10.1186/s1305 4-015-0756-5.

4. Tardella M, Di Carlo M, Carotti M, Filippucci E, Grassi W, Salaffi F. Ultrasound B-lines in the evaluation of interstitial lung disease in patients with systemic sclerosis: cut-off point definition for the presence of significant pulmonary fibrosis. Medicine. 2018;97:18(e0566). https://doi.org/10.1097/MD.0000000000 010566 .

Publisher's Note Springer Nature remains neutral with regard to jurisdictional claims in published maps and institutional affiliations. 